\title{
AN UNCERTAINTY PRINCIPLE LIKE HARDY'S THEOREM FOR NILPOTENT LIE GROUPS
}

\author{
AJAY KUMAR and CHET RAJ BHATTA \\ Dedicated to Eberhard Kaniuth on his 65th birthday
}

(Received 17 May 2002; revised 2 April 2003)

Communicated by A. H. Dooley

\begin{abstract}
We extend an uncertainty principle due to Cowling and Price to threadlike nilpotent Lie groups. This uncertainty principle is a generalization of a classical result due to Hardy. We are thus extending earlier work on $\mathbb{R}^{n}$ and Heisenberg groups.
\end{abstract}

2000 Mathematics subject classification: primary 22E25; secondary 43A30.

Keywords and phrases: uncertainty principle, nilpotent Lie group, Fourier transform, Hilbert-Schmidt norm.

\section{Introduction}

A classical theorem of Hardy [6] on Fourier transform pairs says that a non zero function $f$ on the real line $\mathbb{R}$ and its Fourier transform $\hat{f}$ cannot both be very rapidly decreasing. More precisely, let the Fourier transform be defined by

$$
\hat{f}(y)=\int_{\mathbb{R}} f(x) e^{-2 x i x y} d x, \quad y \in \mathbb{R} .
$$

Hardy's theorem says that if $|f(x)| \leq C e^{-\alpha \pi x^{2}}$ for all $x \in \mathbb{R}$ and $|\hat{f}(y)| \leq C e^{-\beta \pi y^{2}}$ for all $y \in \mathbb{R}$ with $\alpha \beta>1$ then $f=0$ a.e. For a proof see [6] or [4, Theorem 3.2]. The following is a generalization of this theorem due to Cowling and Price [3].

The second author is supported by University Grants Commission under foreign national scheme at the University of Delhi.

(C) 2004 Australian Mathematical Society $1446-7887 / 04 \$ A 2.00+0.00$ 
THEOREM (Cowling and Price). Let $f: \mathbb{R} \rightarrow \mathbb{C}$ be measurable and

(i) $\left\|e_{a} f\right\|_{L^{\rho}(R)}<\infty$,

(ii) $\left\|e_{b} \hat{f}\right\|_{L^{q}(\mathbb{R})}<\infty$,

where $a, b>0, e_{k}(x)=e^{k \pi x^{2}}$ and $1 \leq \min (p, q)<\infty$. If $a b \geq 1$, then $f=0$ almost everywhere. If $a b<1$, then there exist infinitely many linearly independent functions satisfying (i) and (ii).

An analogue of the Cowling-Price Theorem has been proved in [1] for Euclidean spaces, the Heisenberg group $\mathbb{H}_{n}$ and the Euclidean motion group of the plane. In this paper we concern ourselves with results of this kind on certain nilpotent Lie groups, thereby considerably extending the results for $\mathbb{R}^{n}$ and $\mathbb{H}_{n}$.

\section{Threadlike nilpotent Lie groups}

For $n \geq 3$, let $g_{n}$ be the $n$-dimensional real nilpotent Lie algebra with basis $X_{1}, X_{2}, \ldots, X_{n}$ and non trivial Lie brackets $\left[X_{n}, X_{n-1}\right]=X_{n-2}, \ldots,\left[X_{n}, X_{2}\right]=X_{1}$. Here $g_{n}$ is a $(n-1)$-step nilpotent and is a semi-direct product of $\mathbb{R} X_{n}$ and the abelian ideal $\sum_{j=1}^{n-1} \mathbb{R} X_{j}$. Note that $\mathbf{g}_{3}$ is the Heisenberg Lie algebra. Let $G_{n}=\exp \mathbf{g}_{n}$.

For $\xi=\sum_{j=1}^{n-1} \xi_{j} X_{j}^{*} \in \mathbf{g}_{n}^{*}$, the coadjoint action of $G_{n}$ is given by

$$
A d^{*}\left(e^{t X_{n}}\right) \xi=\sum_{j=1}^{n-1} P_{j}(\xi, t) X_{j}^{*},
$$

where, for $1 \leq j \leq n-1, P_{j}(\xi, t)$ is the polynomial in $t$ defined by

$$
P_{j}(\xi, t)=\sum_{k=1}^{j-1}(1 / k !)(-1)^{k} t^{k} \xi_{j-k} .
$$

The orbit of $\xi$ is generic with respect to the basis $\left\{X_{1}^{*}, X_{2}^{*}, \ldots, X_{n}^{*}\right\}$ if and only if $\xi_{1} \neq 0$, and the jumping indices are 2 to $n$; see [2] for details. The cross section $X_{\xi_{1}}$ for the set of generic orbits is given by

$$
X_{\xi_{1}}=\left\{\xi=\left(\xi_{1}, 0, \xi_{3}, \ldots, \xi_{n-1}, 0\right): \xi_{i} \in \mathbb{R}, \xi_{1} \neq 0\right\} .
$$

For $\xi \in \mathbf{g}_{n}^{*}$, let $\pi_{\xi}$ denote the irreducible representation of $G_{n}$ associated with $\xi$. Then the mapping $\xi \rightarrow \pi_{\xi}$ is bijection of $X_{\xi_{1}}$ and the set of all generic irreducible representations. Plancherel measure on $\widehat{G}_{n}$ is supported by these $\pi_{\xi}$.

Denoting by $\mathscr{F}$ the Fourier transform on $\mathbb{R}^{n-1}$, it follows that the Hilbert-Schmidt norm of the operator $\pi_{\xi}(f), f \in L^{1} \cap L^{2}\left(G_{n}\right)$ is given by

$$
\left\|\pi_{\xi}(f)\right\|_{\| S}^{2}=\int_{\mathbb{R}^{2}}\left|\mathscr{F} f\left(P_{1}(\xi, t), \ldots, P_{n-1}(\xi, t), t-s\right)\right|^{2} d s d t
$$


(for details see [2] and [5]).

Given a function $f: G_{n} \rightarrow \mathbb{C}$ and $y=\left(y_{2}, \ldots, y_{n}\right) \in \mathbb{R}^{n-1}$, let $f_{y}, f_{y}^{*}: \mathbb{R} \rightarrow \mathbb{C}$ be defined by

$$
f_{y}\left(x_{1}\right)=f\left(e^{x_{1} X_{1}+\sum_{j=2}^{n} y_{j} x_{j}}\right)
$$

and $f_{y}^{*}\left(x_{1}\right)=\overline{f_{y}\left(-x_{1}\right)}$.

The following lemma is proved in [7, Section 2 and Section 3].

LEMMA 1. Let $f: G_{n} \rightarrow \mathbb{C}$ be a measurable function such that $|f(x)| \leq c e^{-a \pi\|x\|^{2}}$ for some $a, c>0$ and all $x \in G_{n}$. Let $g: \mathbb{R} \rightarrow \mathbb{C}$ be defined by

$$
g\left(x_{1}\right)=\int_{\mathbb{R}^{n-1}} f_{y} * f_{y}^{*}\left(x_{1}\right) d y
$$

Then $\left|g\left(x_{1}\right)\right| \leq C e^{-a \pi x_{1}^{2} / 2}$ for some $C>0$ and all $x_{1} \in \mathbb{R}$ and

$$
\hat{g}\left(\xi_{1}\right)=\left|\xi_{1}\right| \int_{\mathbb{R}^{n-3}}\left\|\pi_{\xi}(f)\right\|_{H S}^{2} d \xi_{3} \cdots d \xi_{n-1}
$$

THEOREM 2. Let $a, b$ and $q$ be real numbers such that $a, b>0$ and $q \geq 2$. Let $f: G_{n} \rightarrow \mathbb{C}$ be a measurable function and suppose that $f$ satisfies:

(i) $|f(x)| \leq C e^{-a \pi\|x\|^{2}}$ for some $C>0$ and all $x \in G_{n}$.

(ii) $\int_{\mathbb{R}^{n-2}}\left|\xi_{1}\right| e^{b q \pi\|\xi\|^{2}}\left\|\pi_{\xi}(f)\right\|_{H S}^{q} d \xi_{1} d \xi_{3} \cdots d \xi_{n-1}<\infty$.

Then the following hold:

(1) If $q=2$ and $a b \geq 1$, then $f=0$ a.e.

(2) If $q>2$ and $a b>1$, then $f=0$ a.e.

Proof. For $\alpha \in \mathbb{R}$, let $e_{\alpha}: \mathbb{R} \rightarrow \mathbb{B}$ denote the function $e_{\alpha}(t)=e^{\alpha \pi t^{2}}$. Let $g: \mathbb{R} \rightarrow \mathbb{C}$ be defined as in Lemma 1. We apply the Cowling-Price Theorem [3] to conclude that $g=0$. Then Lemma 1 shows that $\pi_{\xi}(f)=0$ for almost all $\xi \in \mathbb{R}^{n-2}$, whence $f=0$ a.e.

For $q=2$ by hypothesis (ii),

$$
\left\|e_{2 b} \hat{g}\right\|_{1}=\int_{\mathbb{R}} e_{2 b}\left(\xi_{1}\right)\left(\int_{\mathbb{R}^{n-3}}\left|\xi_{1}\right|\left\|\pi_{\xi}(f)\right\|_{H S}^{2} d \xi_{3} \cdots d \xi_{n-1}\right) d \xi_{1}<\infty
$$

Since $\left|g\left(x_{1}\right)\right| \leq C e^{-a \pi x_{1}^{2} / 2}$ by Lemma 1 and $a b \geq 1$ so the Cowling-Price Theorem yields $g=0$. 
For $q>2$ and $a b>1$, choose $\epsilon>0$ such that $a b^{\prime}>1, b^{\prime}=b-\epsilon$. Then for $\xi^{\prime}=\left(\xi_{3}, \ldots, \xi_{n-1}\right)$, we have

$$
\begin{aligned}
\left\|e_{2 b^{\prime}} \hat{g}\right\|_{q / 2}^{q / 2} & =\int_{\mathbb{R}} e_{b^{\prime} q}\left(\xi_{1}\right)\left|\hat{g}\left(\xi_{1}\right)\right|^{q / 2} d \xi_{1} \\
& =\int_{\mathbb{R}} e_{b^{\prime} q}\left(\xi_{1}\right)\left(\int_{\mathbb{R}^{n-3}}\left|\xi_{1}\right|\left\|\pi_{\xi}(f)\right\|_{H S}^{2} d \xi^{\prime}\right)^{q / 2} d \xi_{1} \\
& \leq \int_{\mathbb{R}} e_{b^{\prime} q}\left(\xi_{1}\right)\left|\xi_{1}\right|^{q / 2}\left(\int_{\mathbb{R}^{n-3}} e_{2 b^{\prime}}\left(\left\|\xi^{\prime}\right\|\right)\left\|\pi_{\xi}(f)\right\|_{H S}^{2} d \xi^{\prime}\right)^{q / 2} d \xi_{1} \\
& =\int_{\mathbb{R}} e_{b^{\prime} q}\left(\xi_{1}\right)\left|\xi_{1}\right|^{q / 2}\left(\int_{\mathbb{R}^{n-3}} e_{2 b}\left(\left\|\xi^{\prime}\right\|\right)\left\|\pi_{\xi}(f)\right\|_{H S}^{2} e_{-2 \epsilon}\left(\left\|\xi^{\prime}\right\|\right) d \xi^{\prime}\right)^{q / 2} d \xi_{1} .
\end{aligned}
$$

Applying Hölder's inequality with $q / 2$ and $q /(q-2)$ we obtain

$$
\begin{aligned}
\left\|e_{2 b} \hat{g}\right\|_{q / 2}^{q / 2} \leq & \int_{\mathbb{R}}\left(e_{b^{\prime} q}\left(\xi_{1}\right)\left|\xi_{1}\right|^{q / 2}\left(\int_{\mathbb{R}^{n-3}} e_{-(2 \epsilon q) /(q-2)}\left(\left\|\xi^{\prime}\right\|\right) d \xi^{\prime}\right)^{(q / 2)-1}\right. \\
& \left.\times \int_{\mathbb{R}^{n-3}} e_{b q}\left(\left\|\xi^{\prime}\right\|\right)\left\|\pi_{\xi}(f)\right\|_{H S}^{q} d \xi^{\prime}\right) d \xi_{1} \\
\leq & K_{1} \int_{\mathbb{R}}\left(e_{q b}\left(\xi_{1}\right)\left(e_{-q \epsilon}\left(\xi_{1}\right)\left|\xi_{1}\right|^{(q / 2)-1}\right)\left|\xi_{1}\right|\right. \\
& \times\left(\int_{\mathbb{R}^{n-3}} e_{b q}\left(\left\|\xi^{\prime}\right\|\right)\left\|\pi_{\xi}(f)\right\|_{H S}^{q} d \xi^{\prime}\right) d \xi_{1} \\
\leq & K \int_{\mathbb{R}^{n-2}} e_{b q}(\|\xi\|)\left\|\pi_{\xi}(f)\right\|_{H S}^{q}\left|\xi_{1}\right| d \xi<\infty
\end{aligned}
$$

for certain positive constants $K_{1}$ and $K$. Thus $g=0$ by the Cowling-Price Theorem.

REMARK 3. If the formula (*) in Lemma 1 reduces to $\hat{g}\left(\xi_{1}\right)=\left|\xi_{1}\right|\left\|\pi_{\xi}(f)\right\|_{H S}^{2}$ for some $G_{n}$, then for $1 \leq q<2$ and $a b \geq 2$ along with the hypothesis in Theorem 2 implies that $f=0$ a.e. The proof can be given as in [1, Theorem 2.1]. The above condition is satisfied if $G_{n}=G_{3}, G_{5,1}, G_{5,3}$ and $G_{5,6}$; see [9] for the definitions and structure of these groups.

THEOREM 4. Let $a$ and $b$ be positive real numbers and $1 \leq \min (p, q)<\infty$. Suppose that $f \in L^{1}\left(G_{n}\right) \cap L^{2}\left(G_{n}\right)$ satisfies the following conditions:

(i) $\int_{G_{n}} e^{p a r\|\cdot\|^{2}}|f(x)|^{p} d x<\infty$,

(ii) $\int_{\mathbb{R}^{n-2}}\left|\xi_{1}\right| e^{b \pi q\|\xi\|^{2}}\left\|\pi_{\xi}(f)\right\|_{H S}^{q} d \xi<\infty$.

If $q \geq 2$ and $a b>1$ then $f=0$ a.e. 
ProOF. Easy computations show that when, as before, identifying $G_{n}$ as a set with $\mathbb{R}^{n}$, the product of two elements $y=\left(y_{1}, \ldots, y_{n}\right)$ and $x=\left(x_{1}, \ldots, x_{n}\right)$ of $G_{n}$ is given by $y x=y+x+\sum_{j=1}^{n-2}(1 / j !) y_{n}^{j}\left(x_{j+1}, \ldots, x_{n-1}, 0, \ldots, 0\right)$. For $\|x\| \geq 1$, this implies

$$
\|y x\| \geq\|x\|-\|y\|-\|x\| \sum_{j=1}^{n-2} \frac{1}{j !}\left|y_{n}\right|^{j} \geq\|x\|\left(1-\|y\|-\sum_{j=1}^{n-2} \frac{1}{j !}\|y\|^{j}\right) .
$$

Define $\varphi:(0, \infty) \rightarrow \mathbb{B}$ by $\varphi(\epsilon)=1-\epsilon-\sum_{j=1}^{n-2}\left(\epsilon^{j} / j !\right)$. Thus $\|y x\| \geq\|x\| \varphi(\epsilon)$, whenever $\|x\| \geq 1$ and $\|y\| \leq \epsilon$.

Let $g$ be a continuous function on $G_{n}$ such that $g(y)=g\left(y^{-1}\right)$ for all $y \in G_{n}$ and $g(y)=0$ for all $y$ such that $\|y\| \geq \epsilon$. Since $G_{n}$ is unimodular, for $x \in G_{n}$ such that $\|x\| \geq 1$,

$$
\begin{aligned}
\left(|g| * e_{a}|f|\right)(x) & =\int_{G_{n}}|g(y)| e_{a}(\|y x\|)|f(y x)| d y \\
& \geq \int_{G_{n}}|g(y)| e_{a}(\|x\| \varphi(\epsilon))\left|f\left(y^{-1} x\right)\right| d y \\
& =e_{a}(\|x\| \varphi(\epsilon))(|g| *|f|)(x) .
\end{aligned}
$$

By (i) $e_{a}|f|$ is an $L^{p}$-function and $|g|$ is an $L^{p^{\prime}}$ function $\left(1 / p+1 / p^{\prime}=1\right)$, so $g * e_{a}|f|$ is an $L^{\infty}$ function. Thus with $C=\left\||g| * e_{a}|f|\right\|_{\infty}<\infty$, we have

$$
|g * f(x)| \leq|g| *|f|(x) \leq C e_{-a}(\|x\| \varphi(\epsilon))
$$

for all $x \in G_{n}$ such that $\|x\| \geq 1$. Since $g * f$ is continuous, it follows that for some constant $C>0,|g * f(x)| \leq C e_{-a}(\|x\| \varphi(\epsilon))$ for all $x \in G_{n}$. In addition,

$$
\left\|\pi_{\xi}(g * f)\right\|_{H S} \leq\left\|\pi_{\xi}(g)\right\| \cdot\left\|\pi_{\xi}(f)\right\|_{H S} \leq\|g\|_{1}\left\|\pi_{\xi}(f)\right\|_{H S}
$$

and hence, by hypothesis

$$
\int_{\mathbb{R}^{n-2}}\left|\xi_{1}\right| e_{b q}(\|\xi\|)\left\|\pi_{\xi}(g * f)\right\|_{H S}^{q} d \xi \leq\|g\|_{1}^{q} \int_{\mathbb{R}^{n-2}}\left|\xi_{1}\right| e_{b q}(\|\xi\|)\left\|\pi_{\xi}(f)\right\|_{H S}^{q} d \xi<\infty
$$

Now for $\epsilon>0$ sufficiently small, $a b \varphi(\epsilon)>1$ so by Theorem 2 it follows that $g * f=0$. Taking for $g$ an approximate identity, we conclude that $f=0$ a.e.

The following result follows from Theorem 2, Remark 3 and Theorem 4.

THEOREM 5. If $G_{n}=G_{3}, G_{5,1}, G_{5,3}$ or $G_{5,6}$ and $a, b>0$. Suppose that $p$ and $q$ are such that $1 \leq \min (p, q)<\infty$ and $f \in L^{1} \cap L^{2}\left(G_{n}\right)$ satisfies 
(i) $\int_{\mathbb{R}^{n}} e^{p a \pi\|x\|^{2}}|f(x)|^{p} d x<\infty$ if $p<\infty$ and $|f(x)| \leq C e^{-a \pi\|x\|^{2}}$ if $p=\infty$,

(ii) $\int_{\mathbb{R}^{n-2}}\left|\xi_{1}\right| e^{b \pi q\|\xi\|^{2}}\left\|\pi_{\xi}(f)\right\|_{H S}^{q} d \xi<\infty$ if $q<\infty$ and $\left\|\pi_{\xi}(f)\right\|_{H S} \leq C e^{-b \pi\|\xi\|^{2}}$ if $q=\infty$.

Then the following hold:

(1) If $q \geq 2$ and $a b>1$, then $f=0$ a.e.

(2) If $1 \leq q<2$ and $a b>2$, then $f=0$ a.e.

Let $G=\exp g$ be a simply connected nilpotent Lie group. Let $U$ denote the Zariski open subset of $\mathbf{g}^{*}$ consisting of all elements in generic orbits with respect to the basis $\left\{X_{1}^{*}, \ldots, X_{n}^{*}\right\}[2$, Section 3.1, Theorem 3.1.9]. Let $S$ be the set of jump indices, and set $T=\{1,2, \ldots, n\} \backslash S$ and $\mathbf{g}_{T}^{*}=\sum_{j \in T} \mathbb{R} X_{j}^{*}$.

Then $X=U \cap \mathbf{g}_{T}^{*}$ is a cross-section for the generic orbits and $\left\{\pi_{\xi}: \xi \in X\right\}$ supports the Plancherel measure on $\widehat{G}$.

The following is a generalization of Morgan's Theorem [8] which can be proved using [7, Lemma 2].

THEOREM 6. Let $G=\operatorname{expg}$ be a simply connected nilpotent Lie group. Let $\alpha, \beta$ and $C$ be positive real numbers and suppose that $f: G \rightarrow \mathbb{C}$ is a measurable function such that

(i) $|f(x)| \leq C e^{-\alpha \pi\|x\|^{\prime \prime}}$,

(ii) $\left\|\pi_{\xi}(f)\right\|_{H S} \leq C e^{-\beta \pi\|\xi\|^{4}}$ for all $\xi=\left(\xi_{1}, \xi_{2}, \ldots, \xi_{n}\right) \in X$,

where $p \geq 2,1 / p+1 / q=1$. If $(\alpha p)^{1 / p}(\beta q)^{1 / q}>2$ then $f=0$ a.e.

\section{Acknowledgements}

The first author is indebted to Professor E. Kaniuth, Mathematik Informatik, Universität Paderborn, Germany for many valuable discussions. The authors thank the referee for useful comments and suggestions.

\section{References}

[1] S. C. Bagchi and S. K. Ray, 'Uncertainty principles like Hardy's theorem on some Lie groups', J. Austral. Math. Soc. (Series A) 65 (1998), 289-302.

[2] L. Corwin and F. P. Greenleaf, Representations of nilpotent Lie groups and their applications, Part l. Basic theory and examples (Cambridge University Press, 1990).

[3] M. G. Cowling and J. F. Price. 'Generalizations of Heisenberg inequality' in: Harmonic Analysis (Cartona 1982), Lecture Notes in Math. 992 (Springer, Berlin, 1983) pp. 443-449.

[4] H. Dym and H. P. Mckean, Fourier series and integrals (Academic Press, New York, 1972).

[5] G. B. Folland, A course in abstract harmonic analysis (CRC Press, Boca Raton, 1995). 
[6] G. H. Hardy, 'A theorem concerning Fourier transforms', J. London Math. Soc. 8 (1993), 227-231.

[7] E. Kaniuth and A. Kumar, 'Hardy's theorem for simply connected nilpotent Lie groups', Math. Proc. Cambridge Philos. Soc. 131 (2001), 487-494.

[8] G. W. Morgan, 'A note on Fourier transform', J. London Math. Soc. 9 (1934), 187-192.

[9] O. A. Nielson, Unitary representations and coadjoint orbits of low-dimensional nilpotent Lie groups, Queens Papers in Pure and Appl. Math. (Queen's Univ., Kingston, ON, 1983).

Department of Mathematics

Rajdhani College

(University of Delhi)

Raja Garden, New Delhi - 110015

India

e-mail: ajaykr@bol.net.in
Department of Mathematics

University of Delhi

Delhi - 110007

India

e-mail: crbhatta@yahoo.com 
J. Aust. Math. Soc. 77 (2004) 